\title{
Accuracy and trending abilities of finger plethysmographic blood pressure and cardiac output compared to invasive measurements during caesarean delivery in healthy women: an observational study
}

Ivar N. Omenås', Christian Tronstad ${ }^{2}$ and Leiv Arne Rosseland ${ }^{1,3^{*}}$ (D)

\begin{abstract}
Background: In women presenting for caesarean section under spinal anesthesia, continuous measurement of circulatory aspects, such as blood pressure and cardiac output, is often needed. At present, invasive techniques are used almost exclusively. Reliable non-invasive monitoring would be welcome, as it could be safer, less uncomfortable, and quick and easy to apply. We aimed to evaluate whether a non-invasive, finger plethysmographic device, the ccNexFin monitor, can replace invasively measured blood pressure in the radial artery, and whether cardiac output measurements from this device can be used interchangeably with measurements from the mini-invasive LiDCO monitor currently in use at our institution.

Methods: Simultaneous invasive measurements were compared to ccNexFin in 23 healthy women during elective caesarean section under spinal anesthesia. We used Bland Altman statistics to assess agreement, and polar plot methodology to judge trending abilities with pre-defined limits.

Results: Mean arterial and systolic pressures showed biases (invasive - ccNexFin) of -4.3 and $12.2 \mathrm{mmHg}$, with limits of agreement of $-15.9-7.4$ and $-11.1-35.6$, respectively. The ccNexFin trending abilities were within the suggested limits for mean pressure but insufficient for systolic pressure compared to invasive measurements. Cardiac output had a small bias of $0.2 \mathrm{~L} / \mathrm{min}$, but wide limits of agreement of $-2.6-3.0$. The ccNexFin trending abilities compared to the invasive estimated values (LiDCO) were unsatisfactory.

Conclusions: We consider the ccNexFin monitor to have sufficient accuracy in measuring mean arterial pressure. The limits of agreement for systolic measurements were wider, and the trending ability compared to invasive measurements was outside the recommended limit. The ccNexFin is not reliable for cardiac output measurements or trend in pregnant women for caesarean delivery under spinal anesthesia.
\end{abstract}

Trial registration: Registered May 23, 2013, at ClinicalTrials.gov under number NCT01861132.

Keywords: Blood pressure, Cardiac output, Caesarean section, Validation studies, Bland Altman, Polar plot

\footnotetext{
* Correspondence: I.a.rosseland@medisin.uio.no

'Division of Emergencies and Critical Care, Oslo University Hospital, Oslo,

Norway

${ }^{3}$ Institute of Clinical Medicine, University of Oslo, Oslo, Norway

Full list of author information is available at the end of the article
}

C C The Author(s). 2020 Open Access This article is licensed under a Creative Commons Attribution 4.0 International License, which permits use, sharing, adaptation, distribution and reproduction in any medium or format, as long as you give appropriate credit to the original author(s) and the source, provide a link to the Creative Commons licence, and indicate if changes were made. The images or other third party material in this article are included in the article's Creative Commons licence, unless indicated otherwise in a credit line to the material. If material is not included in the article's Creative Commons licence and your intended use is not permitted by statutory regulation or exceeds the permitted use, you will need to obtain permission directly from the copyright holder. To view a copy of this licence, visit http://creativecommons.org/licenses/by/4.0/ The Creative Commons Public Domain Dedication waiver (http://creativecommons.org/publicdomain/zero/1.0/) applies to the data made available in this article, unless otherwise stated in a credit line to the data. 


\section{Background}

In a normal pregnancy, large alterations occur in the circulatory physiology. Blood volume, stroke volume, heart rate, and cardiac output (CO) increase, where blood pressure (BP) and peripheral resistance decrease [1]. During caesarean section (CS), both spinal anesthesia and oxytocin administration can precipitate severe drops in peripheral arterial resistance and BP. Vasopressors and inotropes are administered to counteract the changes in $\mathrm{BP}$ and $\mathrm{CO}$ [2]. Perioperatively, BP is usually measured intermittently using oscillatory devices, and changes of short duration are mostly unnoticed, even if substantial. In healthy parturients, changes in $\mathrm{BP}$ and $\mathrm{CO}$ are usually well tolerated and easily corrected, but in patients with pre-eclampsia or cardiac disease, close monitoring of hemodynamic variables is necessary to prevent harm to the mother or fetus. Cardiac disease often goes undiagnosed in pregnant women; of maternal deaths from a cardiac cause in the UK and Ireland in 2009-2014, 77\% did not have a known pre-existing cardiac condition [3].

Anesthetists are trained to observe and interpret heartbeat-to-heartbeat variables and often need to see trends and changes over time. In our department, the current standard tool for continuous cardiovascular monitoring is the LiDCOplus monitor (LiDCO Ltd., London, UK). LiDCO offers continuous data on BP, peripheral resistance, and $\mathrm{CO}$, among other variables. However, the monitor requires intra-arterial access and is reserved for delivery in high-risk pregnancies.

It would be of great advantage for research and clinical monitoring of pregnant women if detailed and reliable continuous measurements could be obtained using noninvasive technologies. Advanced monitoring could be established more quickly and easily, and with less risk. For example, the ccNexFin monitor (NexFin Systems, BMEYE, Amsterdan, NL) is non-invasive and easy to apply. There is, however, currently insufficient evidence that non-invasive monitors are reliable for pregnant women.

The objectives of the present study were to assess the agreement and trending abilities for systolic and mean arterial BP between ccNexFin and invasive BP measures, assess the agreement and trending abilities for $\mathrm{CO}$ between the ccNexFin and LiDCO monitors, and determine if ccNexFin can replace invasive measurements of $\mathrm{BP}$ and $\mathrm{CO}$ during $\mathrm{CS}$ under spinal anesthesia in healthy, pregnant women. The expected outcome was that the non-invasive measurements and trending abilities of $\mathrm{BP}$ are reliable compared to invasive measurements, but we expected $\mathrm{CO}$ to be less reliable.

\section{Methods}

\section{Ethics approval}

The study was approved by the Regional Committee for Medical and Health Research Ethics, Southern Norway
(REC ID: 2012/1155 approved 01/02/2013). All participants provided written informed consent. The study followed the Declaration of Helsinki and was conducted according to Good Clinical Practice.

\section{Patient population}

Healthy, non-smoking, normotensive women with singleton pregnancies scheduled for elective caesarean delivery under spinal anesthesia were asked to participate in this study. Participants were recruited in collaboration with "The Placenta Project" (REC ID: 2011/ 2419) and, for this subset of the study population, a common information leaflet was developed. The information was released during the recruitment period and included detailed information about the anticipated pain during arterial cannulation and the extra time needed when establishing both invasive and non-invasive monitoring. As part of the study protocol for "The Placenta Project", all participants received an intra-arterial line used for blood sampling at delivery. The protocol for The Placenta Project and description of the entire recruitment period was published previously [4]. Exclusion criteria were considerable pre-existing morbidity, pregnancy complications, contractions prior to scheduled Csection, and prior Raynaud phenomena, as this is not compatible with use of the ccNexFin monitor. Two women with hypothyroidism, each supplemented with a low dose of L-thyroxine (50 and $75 \mu \mathrm{g}$ daily) and one woman with mild asthma and occasional use of salbutamol (not taken in the days prior to participation in the study) were included in the study. The inclusion period was from May 2013 to January 2014. Demographics of the study population are given in Table 1 .

\section{Monitoring devices}

The LiDCOplus monitor (LiDCO Ltd., London, UK, version 4.02.95) is in routine clinical use and has documented accuracy and trending abilities [5]. This monitor provides information about circulatory changes from heartbeat to heartbeat and is used when advanced monitoring is indicated, such as during major surgery, or during interventions on patients with circulatory disorders. Mathematical analysis of the intra-arterial pressure curve is performed with pulse power analysis using the built-in software PulseCO. The LiDCO monitor estimates many

Table 1 Patient demographics

\begin{tabular}{lll}
\hline & Mean (SD) & Range \\
\hline Age, yr.mo & $35.10(3.2)$ & $29.6-42.7$ \\
Height, cm & $167(5.0)$ & $160-180$ \\
Weight before pregnancy, kg & $64.0(10.7)$ & $50-91$ \\
Weight at delivery, kg & $78.9(12.3)$ & $60-105$ \\
Length of pregnancy, days & $275(6.5)$ & $260-292$ \\
\hline
\end{tabular}


aspects of the circulation and can be used with or without lithium dilution calibration. In this study, we used calibrated $\mathrm{CO}$, aiming for optimal accuracy. This technology has been used in studies of healthy women and pregnant women with heart disease [6], and is the standard method for perioperative monitoring of preeclamptic women at our institution. In the comparison of $\mathrm{CO}$ methods, $\mathrm{LiDCO}$ serves as the reference.

The ccNexfin monitor (NexFin Systems, BMEYE, Amsterdan, NL, version 1.9.0.1001) is based on the principle of the unloaded vascular wall [7], the Physiocal criteria [8], and a generalized waveform filter to reconstruct brachial pressure from finger pressure [9]. An inflatable cuff is placed around one of the three middle fingers of either hand. An integrated plethysmograph measures the volume of blood under the cuff using an infrared light source and a photosensor. The monitor initially determines a set point for the finger cuff pressure at which most of the venous blood is displaced and the arterial diameter is reduced to no more than $50 \%$ of the expanded diameter. The set point is intermittently calibrated according to the Physiocal criteria to account for changes in the vascular state of the finger. The cuff pressure is continuously adjusted to counter the varying intra-arterial BP, keeping the signal from the photosensor and, consequently, the blood volume and arterial diameter under the cuff constant. This way, the artery wall is said to be unloaded, transmural pressure is zero, and the pressure in the cuff represents the intra-arterial pressure. The measured finger BP is transformed to reflect brachial BP. The $\mathrm{CO}$ calculations in the ccNexFin are based on pulse contour analysis of the derived arterial pressure curve. The monitor is designed to work without external calibration. Our research group has been involved in several projects using finger plethysmographic monitor technology, including the largest population-based study ever utilizing this technology, The Tromsø Study [10].

\section{Study design}

In this prospective observational study, a 20G BD arterial cannula (Becton Dickinson Infusion Therapy Systems, Inc., Utah, USA) was placed in the radial artery after skin infiltration with lidocaine $(5-10 \mathrm{mg})$. The cannula was connected to a Siemens Dräger Infinity Gamma XL hemodynamic monitor (Drägerwerk AG \& Co. KgaA, Lübeck, Germany) via a Codan X-trans pressure transducer (CODAN pvb Critical Care GmbH, Forstinning, Germany) and the signal calibrated according to standard departmental procedures. Peripheral IV catheters were placed on both arms.

Intra-arterial BP data was passed through to the LiDCOplus monitor. Invasive heart rate $\left(\mathrm{HR}_{\text {inv }}\right)$, invasive systolic arterial pressure $\left(\mathrm{SAP}_{\text {inv }}\right)$, and invasive mean arterial pressure $\left(\mathrm{MAP}_{\text {inv }}\right)$ were recorded at a rate of one sample per heartbeat. The $\mathrm{CO}_{\mathrm{LiDCO}}$ estimated by PulseCO was also recorded. A single point calibration of $\mathrm{CO}$ was performed. The ccNexFin monitor was applied to one of the three middle fingers on the same arm as the intra-arterial cannula and corresponding variables $\left(\mathrm{HR}_{\text {nex }}, \mathrm{SAP}_{\text {nex }}, \mathrm{MAP}_{\text {nex }}\right.$, and $\left.\mathrm{CO}_{\text {nex }}\right)$ recorded.

While sitting on the operating table, the subjects received spinal anesthesia with bupivacaine $(10 \mathrm{mg})$ and fentanyl $(20 \mu \mathrm{g})$ using a $27 \mathrm{G}$ pencil point needle. Coloading with intravenous $0.9 \% \mathrm{NaCl}(1000 \mathrm{~mL})$ was started. The parturients were then placed in the supine position with a left lateral tilt using a wedge under the right hip. Immediately after injection of the drugs, an intravenous bolus of phenylephrine $(25-50 \mu \mathrm{g})$ was given, followed by an infusion starting at $0.25 \mu \mathrm{g} / \mathrm{kg} / \mathrm{min}$ and titrated according to invasive BP, aiming for a stable $\mathrm{SAP}_{\text {inv }}>90 \mathrm{mmHg}$.

\section{Data recording}

To acquire synchronous sampling from both monitors, measurements were sampled in real time by the same computer. Samples were acquired through the RS232 port of the LiDCO monitor and the analog output from the ccNexFin monitor using a data acquisition card and software from National Instruments. This setup was evaluated for electrical safety and approved by the appointed committee at Oslo University Hospital.

Time-stamped data for inter-beat interval (IBI), SAP, MAP, and CO from both monitoring devices was recorded to a single dataset per subject, one sample per heartbeat, using software developed in-house using $\mathrm{Na}$ tional Instruments LabVIEW ${ }^{\circ}$. Events were marked in real time and saved to a file using the same software.

Due to subject movement following spinal anesthesia, placement of a hip wedge, and adjustment of the arterial pressure transducer and the ccNexFin heart reference system, we considered data from the first $2 \mathrm{~min}$ after spinal anesthesia as unreliable. The arterial line was used for blood sampling just prior to delivery, causing a pause in our registration, and following delivery there was again more subject movement causing unreliable data. It is also the experience of our group that the LiDCO needs recalibration after delivery [11]. We included the data between 2 and $12 \mathrm{~min}$ after spinal anesthesia for our calculations.

\section{Statistical analysis}

Due to differences in processing time between the two monitors, the LiDCO samples were ahead of the ccNexFin samples, by approximately two heartbeats on average, though they were sampled synchronously from the outputs of the monitors. For each session, this difference was adjusted by calculating the lag using a cross- 
covariance analysis of the IBI time-series, which are assumed to be equal between monitors, and then shifting the ccNexFin recording ahead by the calculated lag in order to align the recordings for comparison at equivalent beats. This was performed in Matlab R2014b (Mathworks, Nantick, Massachusetts, USA).

Artefacts were reduced using a previously published method for detecting and removing outliers in continuous BP and CO recordings [12]. Data points for statistical analysis were constructed with 1-min intervals by averaging data over the first $10 \mathrm{~s}$ of each minute.

Methods were compared using Matlab and Stata v15 (Statacorp LLC, College Station, Texas, USA). We used the method first described by Bland and Altman to investigate the agreement of the ccNexFin monitor with invasive $\mathrm{BP}$ and LiDCO CO measurements [13, 14]. Early versions of this method did not sufficiently consider the structure of the data and could produce too narrow limits of agreement, and too narrow confidence intervals, with repeated measurements per subject. We calculated limits of agreement based on the repeated observations method as described by Zou [15]. Confidence intervals for the limits of agreement were calculated using the MOVER algorithm [15]. This method is preferred when the true value varies, when there is a different number of measurements from each subject, and when the between-subject variance is large with respect to the within-subject variance. The MOVER method also allows the construction of asymmetric CIs. Diagnostic plots suggested by Bland and Altman were inspected to check for underlying assumptions. The high number of repeated measurements increases the information and the statistical power. However, sample size was decided without a formal power calculation.

Polar plots were used to assess trending abilities for both BP and CO [16]. As suggested by Critchley [16], the smallest changes were considered to most likely represent noise and were excluded from the Polar plot analysis. Data points with an average change from the previous measurement of more than $5 \mathrm{mmHg}$ for $\mathrm{BP}$ or $0.5 \mathrm{~L} / \mathrm{min}$ for $\mathrm{CO}$ were included.

\section{Results}

Of 63 subjects approached to participate in the study, 45 agreed to participate. Of these women, 7 went into labor prior to the CS, and study personnel were not available at the time of the CS for 12. Another two women were excluded due to technical problems with the recording equipment, and in one woman we did not succeed in placing an intra-arterial line. This left us with 23 participants eligible for BP analysis. Two additional women were excluded from the analysis of $\mathrm{CO}$ due to unsuccessful calibration of the LiDCO monitor (Fig. 1).
Mean (SD) time from spinal anesthesia to blood sampling was 16.1 (5.5) minutes, with values ranging from 5.6 and $31.7 \mathrm{~min}$. We had some missing data, mostly due to delivery sooner than $12 \mathrm{~min}$ after spinal anesthesia, or misalignment of the ccNexFin heart reference system. For the BP analysis, 209 of a theoretical maximum of 230 data points remained (90.9\%). For the 21 participants included in the $\mathrm{CO}$ analysis, 187 of 210 possible data points remained (89.0\%).

The Bland Altman plots and Polar plots are shown in Fig. 2, and the limits of agreement and Polar plot analyses are shown in Table 2.

\section{Discussion}

Even though the anesthetist aims to keep the patient as stable as possible during caesarean delivery, rapid and major fluctuations in $\mathrm{BP}$ and $\mathrm{CO}$ are common. Both spinal anesthesia and injections of oxytocin result in sudden drops in the peripheral resistance, with decreases in BP and concomitant increases in CO $[2,17]$. Standard clinical practice is to administer fluids and vasopressors [18]. Surgery, bleeding, anxiety, and discomfort also affect the cardiovascular system. Thus, this clinical setting is challenging for any monitoring device, especially regarding trending abilities. Could inclusion of parturients with pre-eclampsia or cardiac diseases have increased the hemodynamic challenge and added value to the evaluation? Pre-eclamptic patients tolerate spinal anesthesia with less of a change in BP and $\mathrm{CO}$ due to peripheral vasoconstriction [19]. Parturients with cardiac disease are another group in which invasive monitoring is indicated and part of standard care in our hospital. The patients usually receive spinal anesthesia, and vasodilation has to be carefully managed throughout the surgery. The hemodynamic response varies a lot depending on cardiac diagnosis and function [6]. Collecting a large sample of patients with invasive monitoring in obstetric anesthesia is challenging. Spinal anesthesia-induced vasodilation and hypotension is common and has a major impact in healthy parturients, and beat-to-beat monitoring is recommended to titrate prophylaxis and the treatment of severe hypotension. Implementing this into practice is important for clinicians who manage parturients with severe cardiac co-morbidity.

\section{Blood pressure varies by measurement site and modality}

The definition of a gold standard for BP is debatable. Although auscultatory BP, measured on the upper arm, may still be considered the gold standard, it is too slow and impractical for use in the setting of caesarean delivery. For most patients, intermittent oscillometric measurements are sufficient, but in more challenging cases, continuous measurements are needed. These measurements are usually obtained invasively. For invasive measurements, the gold standard may be the BP in the 


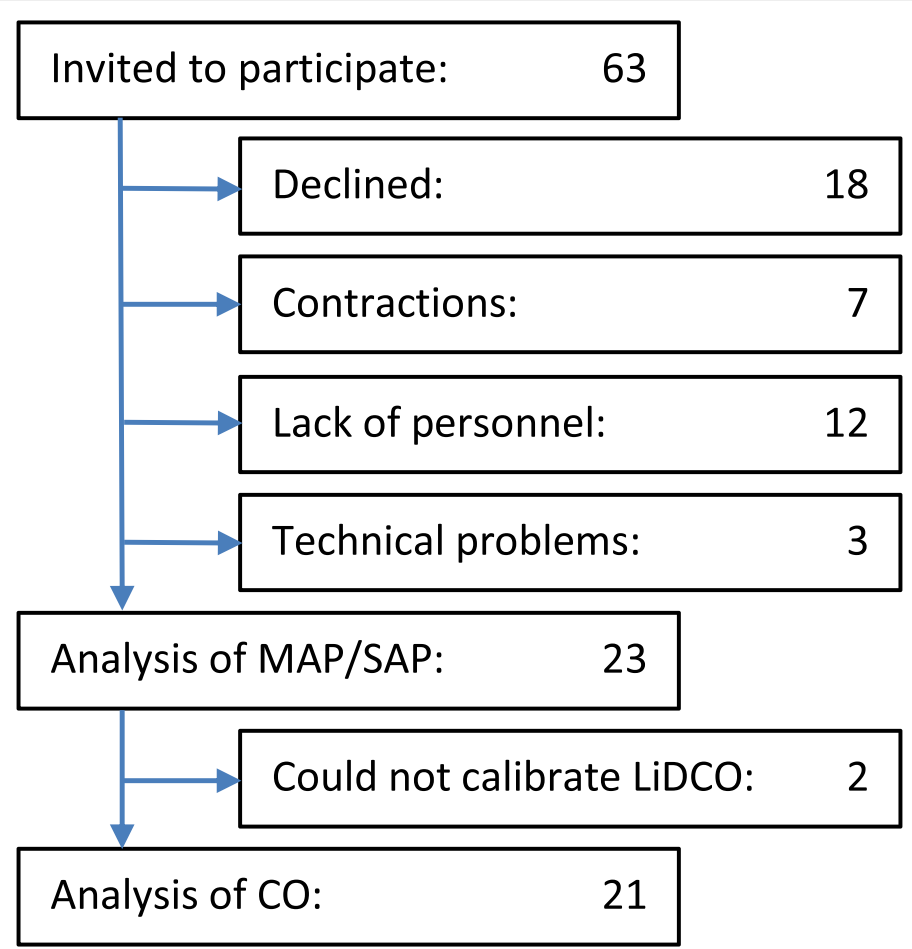

Fig. 1 Flow chart of patient screening, inclusion, and analysis

ascending aorta, but this measurement is not obtainable. The radial artery is the preferred site, as it is conveniently located, easy to cannulate, and the incidence of complications is low [20]. Still, peripherally measured intra-arterial $\mathrm{BP}$ is not equal to the central pressure. The pressure curve is modified as it travels along the branches of the arterial tree by both the elastic properties of the central vessels and reflected waves from the periphery.

Oscillometric non-invasive BP measured in the upper arm underestimates high BP and overestimates low BP, whereas mean pressures are similar compared to intraarterial measurements in the radial artery [21]. In 1990, Gravlee et al. [22], compared intra-arterial BP in the brachial artery measured with four methods of non-invasive BP measurement on the upper arm before, during, and after cardiopulmonary bypass. Averaging overall measurements, they found that the auscultatory method reported lower systolic, but higher mean and diastolic pressures than the invasive measurements, whereas the oscillometric method reported an equal systolic and higher mean and diastolic values compared to invasive measurements. Judging from the graphs, the same is true when considering the first two measurements (i.e. before open chest surgery) separately [22].

In 1951, Wood et al. simultaneously measured the BP in radial and brachial arteries in 17 healthy subjects and found that the SAP in the radial artery was $6 \mathrm{mmHg}$ higher, and MAP and DAP slightly lower (2 and 1
$\mathrm{mmHg}$ ), than in the brachial artery. For hypertensive subjects, the difference in systolic pressure was increased [23]. Pauca et al. measured pressures in the radial artery and in the ascending aorta during bypass surgery. They found small differences in MAP and DAP, but greater differences between centrally and peripherally measured SAP, with pressures in the radial artery being $12 \mathrm{mmHg}$ higher on average. Systolic values were also found to have much greater variance than the mean and diastolic values [24].

The ccNexFin measures BP in a finger. The waveform is transformed to approximate invasive brachial BP [9] using a model that relies on measurements and assumptions in a group of 53 men, some healthy, and some with varying degrees of hypertension and cardiovascular disease. We found similar MAP values between the monitors, with ccNexFin reporting values an average $4 \mathrm{~mm}$ higher than the invasive measurements. Systolic values were approximately $12 \mathrm{~mm}$ lower on average. A slightly lower systolic value is expected, as ccNexFin aims to represent a more central pressure, but the difference is larger than can reasonably be explained by this effect. The inaccuracy is small and will likely be of minor importance during caesarean delivery under spinal anesthesia.

\section{Varying definitions of hypotension}

An analysis by Klöhr et al. reviewing definitions of hypotension after spinal anesthesia for caesarean delivery 
A

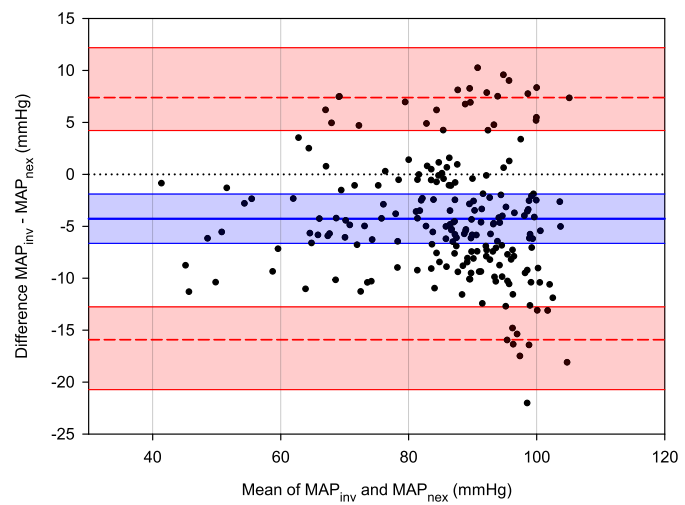

C

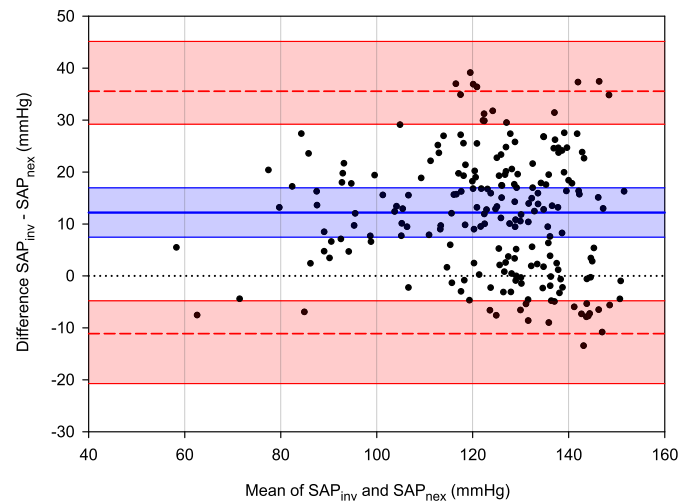

E

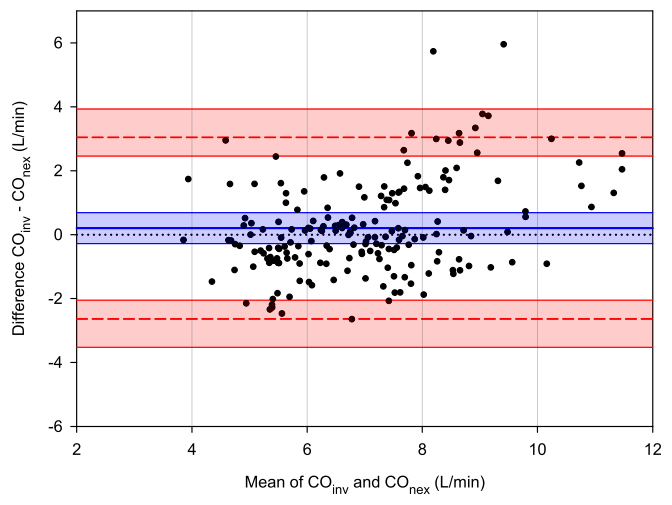

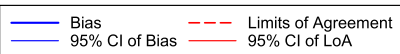

B

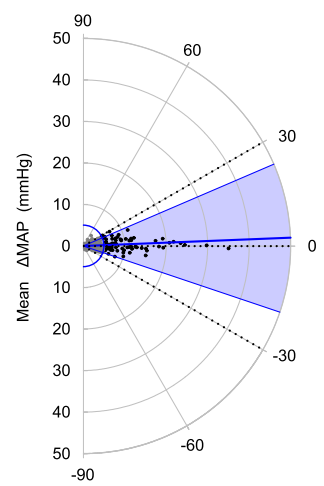

D

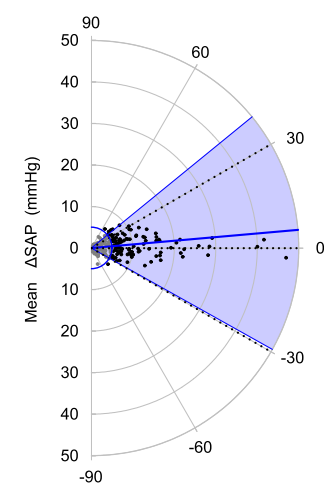

F

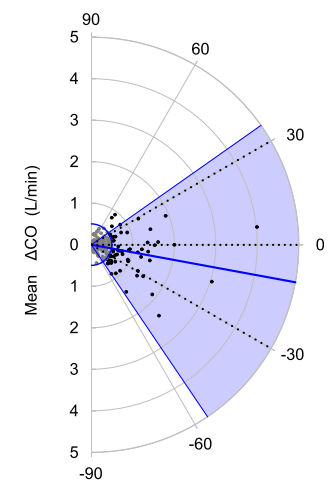

Mean Polar Angle

Fig. 2 Comparison of invasive measurements with ccNexFin. a Mean arterial pressure (MAP) in a Bland Altman plot and b Polar plot. c Systolic arterial pressure (SAP) in a Bland Altman plot and d Polar plot. e Cardiac output (CO) in a Bland Altman plot and f Polar plot

Table 2 Bias (invasive - ccNexFin), limits of agreement (LoA), and Polar plot analyses

\begin{tabular}{lllll}
\hline & Bias $(95 \% \mathrm{Cl})$ & LoA $(95 \% \mathrm{Cl})$ & Mean polar angle (SD) & Radial LoA \\
\hline MAP & $-4.3 \mathrm{mmHg}(-6.6,-1,9)$ & $-15.9(-20.7,-12.8)$ to $7.4(4.2,12.2)$ & $2.3^{\circ}\left(10.0^{\circ}\right)$ & $-18.6^{\circ}, 23.2^{\circ}$ \\
SAP & $12.2 \mathrm{mmHg}(7.5,17.0)$ & $-11.1(-20.7,-4.8)$ to $35.6(29.2,45.2)$ & $5.0^{\circ}\left(17.3^{\circ}\right)$ & $-29.2^{\circ}, 39.2^{\circ}$ \\
CO & $0.2 \mathrm{~L} / \mathrm{min}(-0.3,0.7)$ & $-2.6(-3.5,-2.0)$ to $3.0(2.5,3.9)$ & $-10.4^{\circ}\left(27.2^{\circ}\right)$ & $-55.9^{\circ}, 35.1^{\circ}$ \\
\hline
\end{tabular}


found that, in research, relative limits seem to be more popular than absolute thresholds [25]. This and other papers reference a survey by Burns et al. from 2001 as an argument that anesthetists prefer absolute thresholds in this setting [26], but they actually did not. The authors claimed that a relative rather than an absolute decrease may be more important. They added that, not only the degree of hypotension, but also the duration may be important. Nevertheless, as absolute limits do not require a baseline to be determined, it may be reasonable to assume that this simpler approach would be preferred by many. In a recent consensus document, Kinsella et al. suggested taking a baseline BP measurement before spinal anesthesia using repeated measurements if the BP is not stable, if it is higher than expected, or if the woman is in labor [27]. They recommended aiming for a SAP $\geq 90 \%$ of baseline and to avoid a decrease to $<80 \%$ of baseline. The SAP was also suggested to be a less important variable than MAP as a determinant of organ perfusion. Recommended limits are still based on the SAP, as this has been the primary outcome in most of the available research. MAP is unlikely to be used to define hypotension in this clinical setting without more supportive data [27].

Auscultatory, oscillometric, invasive, and finger plethysmographic techniques use different principles to measure or estimate BP. In addition, measurements are made at different anatomic sites. It is important to be aware of the measurement techniques used in scientific studies, their definitions of safe limits for BP, and to consider the characteristics of these methods compared to the ones being used in clinical practice.

From this perspective, the inaccuracy of ccNexFin compared to invasive arterial pressure has a minor impact, and we recommend finger plethysmographic measurement for clinical use and in research.

\section{Trending abilities for blood pressure}

The degree to which two monitors agree in their ability to track changes is also crucial. For the Polar plot method we applied, Critchley et al. suggest using a limit of $\pm 5^{\circ}$ for angular bias and radial limits of no more than $\pm 30^{\circ}$ for good trending abilities [16]. Only the MAP measurements satisfied these limits. The low angular bias suggests that the monitors were in good calibration, and with radial limits well within $\pm 30^{\circ}$ the ccNexFin exhibits good trending ability compared to intra-arterial measurements. The SAP had an angular bias on the border of the suggested range, but the radial limits were too wide. Taking into consideration the hemodynamic variations typical for caesarean delivery under spinal anesthesia, this is a challenging model, with larger intraindividual variations than in critical care patients. We recommend finger plethysmographic measurements in clinical settings and for research purposes requiring good BP trending ability, such as repeated measurements. In pre-eclampsia or other settings with pregnancy-induced hypertension, invasive measurements should replace the finger plethysmographic method due to the tendency to underestimate SAP.

\section{Cardiac output}

Regarding CO, we did not find sufficient agreement between the monitors. Even though the bias was small, the limits of agreement were wide, more than $\pm 40 \%$ of the mean $\mathrm{CO}$. Both the angular bias and radial limits of agreement were far outside recommended limits, suggesting that ccNexFin cannot reliably track changes in CO. This is consistent with the results in a study comparing this technology to echocardiographic estimates of $\mathrm{CO}$ [28]. Based on the interpretation of the results, we do not recommend using ccNexFin to measure $\mathrm{CO}$ or to monitor $\mathrm{CO}$ trends in the clinic or research in pregnant women.

\section{Limitations}

In this study, we presented the accuracy (bias) and precision (variability) of agreement between two methods. We calculated limits of agreement and assessed the use of ccNexfin against invasive measurements for BP and the LiDCO monitor for CO. As described by Hapfelmaier et al. [29], the precision of agreement partly depends on the precision of measurement (repeatability) of both devices. For example, the limits of agreement will become wider as a consequence of using an imprecise reference technique. This means that the agreement between a new technique and a reference technique needs to be judged in light of the precision that the techniques themselves are able to achieve [29]. In the present study, it was not possible to determine the repeatability (variation around a true value). Obstetric anesthesia is characterized by constantly changing hemodynamics, and repeated measurements during one constant value of $\mathrm{BP}$ or $\mathrm{CO}$ within the experiment is impossible. Thus, determining the precision of measurement of the LiDCO per se and the Nexfin technique per se was not possible.

A total of 21 patients were analyzed and a larger sample could have increased the precision and generalizability. However, it is important to take into consideration the large number of repeated measurements per patient and that the analyses included all of these data points to assess trending abilities.

\section{Conclusion}

We consider the ccNexFin monitor to have sufficient accuracy in measuring MAP. The limits of agreement for 
systolic measurements were wider, and the trending ability compared to invasive measurements was outside the recommended limit. The ccNexFin is not reliable for $\mathrm{CO}$ measurements or trending ability in pregnant women undergoing caesarean delivery under spinal anesthesia.

\section{Abbreviations}

CO: Cardiac output; BP: Blood pressure; HR: Heart rate; SAP: Systolic arterial pressure; MAP: Mean arterial pressure; IBI: Inter-beat interval

\section{Acknowledgements}

The authors thank Dr. Ane M. Holme, Dr. Maia B. Holm, and Dr. Hildegunn Horne for valuable collaboration during data collection.

\section{Authors' contributions}

INO contributed during planning, data collection, analyses, and interpretation, and manuscript preparation. CT contributed during planning, data collection, analyses, and interpretation, and manuscript preparation. LAR is the principle investigator, contributed during planning, approval of the protocol, data collection, analyses, and interpretation, and manuscript preparation. All authors have read and approved the manuscript.

\section{Funding}

Internal funding (Oslo University Hospital) covered all costs including equipment, data management, and work hours.

\section{Availability of data and materials}

The datasets used and analyzed during the current study is available from the corresponding author on reasonable request.

\section{Ethics approval and consent to participate}

The study was approved by the Regional Committee for Medical and Health Research Ethics, Southern Norway (REC ID: 2012/1155 approved 01/02/2013). All participants provided written informed consent. The study followed the Declaration of Helsinki.

\section{Consent for publication}

Not applicable.

\section{Competing interests}

Ivar N. Omenås declares no conflict of interest. Christian Tronstad declares no conflict of interest. Leiv Arne Rosseland declares no conflict of interest

\section{Author details}

'Division of Emergencies and Critical Care, Oslo University Hospital, Oslo, Norway. ${ }^{2}$ Department of Clinical and Biomedical Engineering, Oslo University Hospital, Oslo, Norway. ${ }^{3}$ Institute of Clinical Medicine, University of Oslo, Oslo, Norway.

Received: 19 March 2020 Accepted: 18 June 2020

Published online: 27 June 2020

\section{References}

1. Grindheim G, Estensen M-E, Langesaeter E, Rosseland LA, Toska K. Changes in blood pressure during healthy pregnancy: a longitudinal cohort study. J Hypertens. 2012;30:342-50

2. Langesaeter E, Rosseland L, Stubhaug A. Hemodynamic effects of oxytocin during cesarean delivery. Int J Gynaecol Obstet. 2006;95:46-7.

3. Knight M, Nair M, Tuffnell D, Kenyon S, Shakespeare J, Brocklehurst P, et al. Saving Lives, Improving Mothers' Care - Surveillance of maternal deaths in the UK 2012-14 and lessons learned to inform maternity care from the UK and Ireland. 2016.

4. Holme AM, Holm MB, Roland MCP, Horne H, Michelsen TM, Haugen G, Henriksen, T. The 4-vessel Sampling Approach to Integrative Studies of Human Placental Physiology In Vivo. J Vis Exp. 2017;(126):e55847. https://doi. org/10.3791/55847.

5. Langesaeter E, Gibbs M, Dyer RA. The role of cardiac output monitoring in obstetric anesthesia. Curr Opin Anaesthesiol. 2015;28:247-53.
6. Langesaeter E, Dragsund M, Rosseland L. Regional anaesthesia for a caesarean section in women with cardiac disease: a prospective study. Acta Anaesthesiol Scand. 2010;54:46-54.

7. Penaz J. Photoelectric Measurement of Blood Pressure, Voluma and Flow in the Finger. Digest of the 10th International Conference on Medical and Biological Engineering - 1973 - Dresden; 1973.

8. Wesseling KH, De Wit B, Van der Hoeven GMA, Van Goudoever J, Settels JJ. Physiocal, calibrating finger vascular physiology for Finapres. Homeostasis. 1995;36:67-82.

9. Gizdulich P, Prentza A, Wesseling KH. Models of brachial to finger pulse wave distortion and pressure decrement. Cardiovasc Res. 1997;33:698-705.

10. Olsen RB, Bruehl S, Nielsen CS, Rosseland LA, Eggen AE, Stubhaug A. Chronic pain and cardiovascular stress responses in a general population: the Tromsø study. J Behav Med. 2014;37:1193-201.

11. Langesaeter E. Is it more informative to focus on cardiac output than blood pressure during spinal anesthesia for cesarean delivery in women with severe preeclampsia? Anesthesiology. 2008:108:771-2.

12. Tronstad C, Omenås IN, Rosseland LA. An improved artifact removal algorithm for continuous cardiac output and blood pressure recordings. 2015 37th Annual International Conference of the IEEE Engineering in Medicine and Biology Society (EMBC). New York City: IEEE; 2015. p. 5968-72.

13. Bland JM, Altman DG. Statistical methods for assessing agreement between two methods of clinical measurement. Lancet. 1986;1:307-10.

14. Bland JM, Altman DG. Agreement between methods of measurement with multiple observations per individual. J Biopharm Stat. 2007;17:571-82.

15. Zou GY. Confidence interval estimation for the Bland-Altman limits of agreement with multiple observations per individual. Stat Methods Med Res. 2013;22:630-42.

16. Critchley LA, Yang XX, Lee A. Assessment of trending ability of cardiac output monitors by polar plot methodology. J Cardiothorac Vasc Anesth. 2011;25:536-46

17. Langesæter E, Rosseland LA, Stubhaug A. Continuous invasive blood pressure and cardiac output monitoring during cesarean delivery: a randomized, double-blind comparison of low-dose versus high-dose spinal anesthesia with intravenous phenylephrine or placebo infusion. Anesthesiology. 2008;109:856-63.

18. Fitzgerald JP, Fedoruk KA, Jadin SM, Carvalho B, Halpern SH. Prevention of hypotension after spinal anaesthesia for caesarean section: a systematic review and network meta-analysis of randomised controlled trials. Anaesthesia. 2020;75:109-21.

19. Sharwood-Smith G, Drummond GB. Hypotension in obstetric spinal anaesthesia: a lesson from pre-eclampsia. Br J Anaesth Oxford University Press. 2009:102:291-4.

20. Scheer B, Perel A, Pfeiffer UJ. Clinical review: complications and risk factors of peripheral arterial catheters used for haemodynamic monitoring in anaesthesia and intensive care medicine. Crit Care. 2002; 6:199-204.

21. Wax DB, Lin H-M, Leibowitz AB. Invasive and concomitant noninvasive intraoperative blood pressure monitoring: observed differences in measurements and associated therapeutic interventions. Anesthesiology. 2011;115:973-8.

22. Gravlee GP, Brockschmidt JK. Accuracy of four indirect methods of blood pressure measurement, with hemodynamic correlations. J Clin Monit. 1990; 6:284-98.

23. Wood EH, Fuller J, Clagett OT. Intraluminal pressures recorded simultaneously from different arteries in man. Am J Phys. 1951;167:838-9.

24. Pauca AL, Wallenhaupt SL, Kon ND, Tucker WY. Does radial artery pressure accurately reflect aortic pressure? Chest. 1992;102:1193-8.

25. Klöhr S, Roth R, Hofmann T, Rossaint R, Heesen M. Definitions of hypotension after spinal anaesthesia for caesarean section: literature search and application to parturients. Acta Anaesthesiol Scand. 2010; 54:909-21.

26. Burns SM, Cowan CM, Wilkes RG. Prevention and management of hypotension during spinal anaesthesia for elective caesarean section: a survey of practice. Anaesthesia. 2001;56:794-8.

27. Kinsella SM, Carvalho B, Dyer RA, Fernando R, McDonnell N, Mercier FJ, et al. International consensus statement on the management of hypotension with vasopressors during caesarean section under spinal anaesthesia. Anaesthesia. 2018;73:71-92.

28. Duclos G, Hili A, Resseguier N, Kelway C, Haddam M, Bourgoin A, et al. Clearsight ${ }^{\mathrm{TM}}$ use for haemodynamic monitoring during the third 
trimester of pregnancy - a validation study. Int J Obstet Anesth Elsevier Ltd. 2018;36:85-95.

29. Hapfelmeier A, Cecconi M, Saugel B. Cardiac output method comparison studies: the relation of the precision of agreement and the precision of method. J Clin Monit Comput. 2016;30:149-55.

\section{Publisher's Note}

Springer Nature remains neutral with regard to jurisdictional claims in published maps and institutional affiliations.

Ready to submit your research? Choose BMC and benefit from:

- fast, convenient online submission

- thorough peer review by experienced researchers in your field

- rapid publication on acceptance

- support for research data, including large and complex data types

- gold Open Access which fosters wider collaboration and increased citations

- maximum visibility for your research: over $100 \mathrm{M}$ website views per year

At BMC, research is always in progress. 